\title{
A Framework for Derivative Pricing in the Fractional Black-Scholes Market
}

\author{
Ciprian Necula \\ DOFIN \\ Academy of Economic Studies \\ Bucharest, Romania \\ email: ciprian.necula@fin.ase.ro
}

First draft: September 122007

\begin{abstract}
The aim of this paper is to develop a framework for evaluating derivatives if the underlying of the derivative contract is supposed to be driven by a fractional Brownian motion with Hurst parameter greater than 0.5. For this purpose we first prove some results regarding the quasi-conditional expectation, especially the behavior to a Girsanov transform. We obtain the risk-neutral valuation formula and the fundamental evaluation equation in the case of the fractional Black-Scholes market.
\end{abstract}

Keywords: fractional Brownian motion, fractional Black-Scholes market, quasiconditional expectation, mathematical finance, contingent claim

JEL Classification: C02, C60, G12, G13

AMS Classification: 60H05, 60H10, 91B26, 91B26, 91B70 


\section{INTRODUCTION}

The fractional Brownian motion ( $\mathrm{fBm}$ ) with Hurst parameter $H$ $(0<H<1)$ is the continuous Gaussian process $\left\{B_{H}(t), t \in \mathbb{R}\right\}, B_{H}(t)=0$ with mean $E\left[B_{H}(t)\right]=0$ and whose covariance is given by:

$$
C_{H}(t, s)=E\left[B_{H}(t) B_{H}(t)\right]=\frac{1}{2}\left\{|t|^{2 H}+|s|^{2 H}-|t-s|^{2 H}\right\}
$$

If $H=\frac{1}{2}$ then $B_{H}(t)$ coincides with the standard Brownian motion $B(t)$.

The fractional Brownian motion is a self-similar process meaning that for any $\alpha>0 \quad B_{H}(\alpha t)$ has the same law as $\alpha^{H} B_{H}(t)$.

The parameter $H$ determines the sign of the covariance of the future and past increments. This covariance is positive when $H>\frac{1}{2}$, zero when $H=\frac{1}{2}$ (i.e. the classical Brownian motion ) and negative when $H<\frac{1}{2}$.

Another property of the fractional Brownian motion is that for $H>\frac{1}{2}$ it has long-range dependence in the sense that if we put

$$
r(n)=\operatorname{Cov}\left(B_{H}(1), B_{H}(n+1)-B_{H}(n)\right)
$$

then

$$
\sum_{n=1}^{\infty} r(n)=\infty
$$

The self-similarity and long-range dependence properties make the fractional Brownian motion a suitable tool in different applications like mathematical finance. Since for $H \neq \frac{1}{2}$ the fractional Brownian motion is neither a Markov process, nor a semimartingale (see for exemple Rogers, 1997), we cannot use the usual stochastic calculus to analyse it. Worse still, after a pathwise integration theory for fractional Brownian motion was developed by Lin (1995) and Decreusefond and Ustunel (1999), it was proven by Rogers (1997) that the market mathematical models driven by $B_{H}(t)$ could have arbitrage. The fractional Brownian motion was no longer considered fit for mathematical modeling in finance. However after the development by Duncan, Hu and Pasik-Duncan (2000) and $\mathrm{Hu}$ and Oksendal (2003) of a new kind of integral based on the Wick product 
called the fractional Ito integral, it was proved in $\mathrm{Hu}$ and Oksendal (2003) that the corresponding Ito type fractional Black-Schools market has no arbitrage. Equivalent definitions of the fractional Ito integral were introduced by Alos, Mazet and Nualart (2000), Perez-Abreau and Tudor (2002) and Bender (2002). Hu and Oksendal (2003) introduced the concept of quasi-conditional expectation and quasi-martingales. In the same paper a formula for the price of a European option at $t=0$ is derived.

The aim of this paper is to build a framework for evaluating derivatives if the underlying of the derivative contract is supposed to be driven by a fractional Brownian motion with Hurst parameter greater than 0.5.

This paper is organized as follows: in the first section we prove some results regarding the quasi-conditional expectation, especially the behaviour to a Girsanov transform. In the third section we apply these results to obtain the riskneutral valuation formula and the fundamental evaluation equation in the case of the fractional Black-Scholes market. The final section concludes.

\section{EXPECTATION \\ 2. SOME RESULTS REGARDING THE QUASI-CONDITIONAL}

Let $(\Omega, \mathrm{K}, P)$ a probability field such that $B_{H}(t, \omega)$ is a fractional Brownian motion with respect to $P, \mathrm{~F}_{t}^{H}:=\mathrm{B}\left(B_{H}(s), s \leq t\right)$ and $\widetilde{E}_{t}[]$ the quasiconditional expectation with respect to $\mathrm{F}_{t}{ }^{H}$ (definition 4.9 in $\mathrm{Hu}$ and Oksendal, 2003).

THEOREM 2.1. For every $0<t<T$ and $\lambda \in \mathbb{C}$ we have that

$$
\widetilde{E}_{t}\left[e^{\lambda B_{H}(T)}\right]=e^{\lambda B_{H}(t)+\frac{\lambda^{2}}{2}\left(T^{2 H}-t^{2 H}\right)}
$$

Proof:

Consider the fractional differential equation:

$$
d X(t)=\lambda X(t) d B_{H}(t), \quad X(0)=1
$$

The solution of this equation is (Hu and Oksendal, 2003):

$$
X(t)=\exp \left(\lambda B_{H}(t)-\frac{1}{2} \lambda^{2} t^{2 H}\right)
$$

Since 


$$
X(t)=\int_{0}^{t} \lambda X(s) d B_{H}(s)
$$

we know that $X(t)$ is a quasi-martingale (Hu and Oksendal, 2003). So it follows that:

$$
\widetilde{E}_{t}[X(T)]=X(t)
$$

or

$$
\widetilde{E}_{t}\left[e^{\lambda B_{H}(T)}\right]=e^{\lambda B_{H}(t)+\frac{\lambda^{2}}{2}\left(T^{2 H}-t^{2 H}\right)}
$$

q.e.d.

THEOREM 2.2. Let $f: \mathbb{R} \rightarrow \mathbb{R}$ be a function such that $E\left[f\left(B_{H}(T)\right)\right]<\infty$. Then for every $t \leq T$

$$
\widetilde{E}_{t}\left[f\left(B_{H}(T)\right)\right]=\int_{\mathrm{R}} \frac{1}{\sqrt{2 \pi\left(T^{2 H}-t^{2 H}\right)}} \exp \left(-\frac{\left(x-B_{H}(t)\right)^{2}}{2\left(T^{2 H}-t^{2 H}\right)}\right) f(x) d x .
$$

\section{Proof:}

Let $\hat{f}$ be the Fourier transform of $f$ :

$$
\hat{f}(\xi)=\int_{\mathbb{R}} e^{-i x \xi} f(x) d x
$$

Then $f$ is the inverse Fourier transform of $\hat{f}$ :

$$
f(x)=\frac{1}{2 \pi} \int_{\mathbb{R}} e^{i x \xi} \hat{f}(\xi) d \xi
$$

We have that:

$$
f\left(B_{H}(T)\right)=\frac{1}{2 \pi} \int_{\mathbb{R}} e^{i B_{H}(T) \xi} \hat{f}(\xi) d \xi
$$

It follows that:

$$
\widetilde{E}_{t}\left[f\left(B_{H}(T)\right)\right]=\widetilde{E}_{t}\left[\frac{1}{2 \pi} \int_{\mathbb{R}} e^{i \xi B_{H}(T)} \hat{f}(\xi) d \xi\right]
$$




$$
\begin{aligned}
& =\frac{1}{2 \pi} \int_{\mathbb{R}} \widetilde{E}_{t}\left[e^{i \xi B_{H}(T)}\right] \hat{f}(\xi) d \xi \\
& =\frac{1}{2 \pi} \int_{\mathbb{R}} e^{i \xi B_{H}(t)-\frac{\xi^{2}}{2}\left(T^{2 H}-t^{2 H}\right)} \hat{f}(\xi) d \xi \\
& =h\left(B_{H}(t)\right)
\end{aligned}
$$

where $h$ is the inverse Fourier transform of the product between $e^{-\frac{\xi^{2}}{2}\left(T^{2 H}-t^{2 H}\right)}$ and $\hat{f}(\xi)$.

But the first function is the Fourier transform of

$$
n_{t, T}(x)=\frac{1}{\sqrt{2 \pi\left(T^{2 H}-t^{2 H}\right)}} \exp \left(-\frac{x^{2}}{2\left(T^{2 H}-t^{2 H}\right)}\right)
$$

Using the fact that the Fourier transform of a convolution is the product of the Fourier transform of the two functions it follows that

$$
h\left(B_{H}(t)\right)=\int_{\mathbb{R}} n_{t, T}\left(B_{H}(t)-y\right) f(y) d y
$$

COROLLARY 2.3. Let $A \in \mathrm{B}(\mathbb{R})$. Then

$$
\widetilde{E}_{t}\left[\mathbb{1}_{A}\left(B_{H}(T)\right)\right]=\int_{A} \frac{1}{\sqrt{2 \pi\left(T^{2 H}-t^{2 H}\right)}} \exp \left(-\frac{\left(x-B_{H}(t)\right)^{2}}{2\left(T^{2 H}-t^{2 H}\right)}\right) d x
$$

Let $\theta \in \mathbb{R}$. Consider the process

$$
B_{H}^{*}(t)=B_{H}(t)+\theta t^{2 H}=B_{H}(t)+\int_{0}^{t} 2 H \theta \tau^{2 H-1} d \tau, \quad 0 \leq t \leq T
$$

Theorem 3.18 in $\mathrm{Hu}$ and Oksendal (2003) assures us that there is a measure $P^{*}$ such that $B_{H}^{*}(t)$ is a fractional Brownian motion under $P^{*}$.

We will denote $\widetilde{E}_{t}^{*}[\cdot]$ the quasi-conditional expectation with respect to $P^{*}$.

Consider 


$$
Z(t):=\exp \left(-\theta B_{H}(t)-\frac{\theta^{2}}{2} t^{2 H}\right)
$$

THEOREM 2.4. Let $f$ be a function such that $E\left[f\left(B_{H}(T)\right)\right]<\infty$. Then for every $t \leq T$

$$
\widetilde{E}_{t}^{*}\left[f\left(B_{H}(T)\right)\right]=\frac{1}{Z(t)} \widetilde{E}_{t}\left[f\left(B_{H}(T)\right) Z(T)\right]
$$

Proof:

Again we will denote by $\hat{f}$ the Fourier transform of $f$.

We have

$$
\begin{aligned}
\widetilde{E}_{t}\left[f\left(B_{H}(T)\right) Z(T)\right] & =\widetilde{E}_{t}\left[\frac{1}{2 \pi} \int_{R} e^{(i \xi-\theta) B_{H}(T)-\frac{\theta^{2}}{2} T^{2 H}} \hat{f}(\xi) d \xi\right] \\
& =\frac{1}{2 \pi} e^{-\frac{\theta^{2}}{2} T^{2 H}} \int_{R} \widetilde{E}_{t}\left[e^{(i \xi-\theta) B_{H}(T)}\right] \hat{f}(\xi) d \xi \\
& =\frac{1}{2 \pi} e^{-\frac{\theta^{2}}{2} T^{2 H}} \int_{R} e^{(i \xi-\theta) B_{H}(t)+\left(-\frac{\xi^{2}}{2}-i \xi \theta+\frac{\theta^{2}}{2}\right)\left(T^{2 H}-t^{2 H}\right)} \hat{f}(\xi) d \xi \\
& =Z(t) \frac{1}{2 \pi} \int_{R} e^{i \xi B_{H}(t)+\left(-\frac{\xi^{2}}{2}-i \xi \theta\right)\left(T^{2 H}-t^{2 H}\right)} \hat{f}(\xi) d \xi
\end{aligned}
$$

On the other hand

$$
\begin{aligned}
\widetilde{E}_{t}^{*}\left[f\left(B_{H}(T)\right)\right] & =\widetilde{E}_{t}^{*}\left[\frac{1}{2 \pi} \int_{R} e^{i \xi\left(B_{H}^{*}(T)-\theta T^{2 H}\right)} \hat{f}(\xi) d \xi\right] \\
& =\frac{1}{2 \pi} \int_{R} \widetilde{E}_{t}^{*}\left[e^{i \xi B_{H}^{*}(T)}\right] e^{-i \xi \theta T^{2 H}} \hat{f}(\xi) d \xi \\
& =\frac{1}{2 \pi} \int_{R} e^{i \xi B_{H}^{*}(t)-\frac{\xi^{2}}{2}\left(T^{2 H}-t^{2 H}\right)} e^{-i \xi \theta T^{2 H}} \hat{f}(\xi) d \xi \\
& =\frac{1}{2 \pi} \int_{R} e^{i \xi\left(B_{H}(t)+\theta t^{2 H}\right)-\frac{\xi^{2}}{2}\left(T^{2 H}-t^{2 H}\right)} e^{-i \xi \theta T^{2 H}} \hat{f}(\xi) d \xi
\end{aligned}
$$




$$
=\frac{1}{2 \pi} \int_{R} e^{i \xi B_{H}(t)+\left(-\frac{\xi^{2}}{2}-i \xi \theta\right)\left(T^{2 H}-t^{2 H}\right)} \hat{f}(\xi) d \xi
$$

The result follows immediately.

q.e.d.

3. RISK NEUTRAL VALUATION IN THE FRACTIONAL BLACKSCHOLES MARKET

Consider the fractional Black-Scholes market consisting of two investment possibilities:

1.

a money market account:

$$
d M(t)=r M(t) d t, \quad M(0)=1, \quad 0 \leq t \leq T
$$

where $r$ represent the constant riskless interest rate.

2.

a stock whose price satisfies the equation:

$$
d S(t)=\mu S(t) d t+\sigma S(t) d \bar{B}_{H}(t), \quad S(0)=S>0,0 \leq t \leq T
$$

where $\mu, \sigma \neq 0$ are constants and $\bar{B}_{H}(t)$ is a fractional Brownian motion with respect to the market measure.

In $\mathrm{Hu}$ and Oksendal (2003) it was shown that this market does not have arbitrage and is complete, the same properties as the classical Black-Scholes model based on the Brownian motion.

Under the risk-neutral measure (denoted $P$ ) we have that:

$$
d S(t)=r S(t) d t+\sigma S(t) d B_{H}(t), \quad S(0)=S>0,0 \leq t \leq T
$$

where $B_{H}(t)$ is a fractional Brownian motion with respect to $P$.

The solution of this stochastic differential equation is (Hu and Oksendal, 2003):

$$
S(t)=S \exp \left(\sigma B_{H}(t)+r t-\frac{1}{2} \sigma^{2} t^{2 H}\right)
$$

We will denote by $\widetilde{E}_{t}[\cdot]$ the quasi-conditional expectation with respect to the risk-neutral measure.

THEOREM 3.1. (fractional risk-neutral evaluation)

The price at every $t \in[0, T]$ of a bounded $\mathrm{F}_{T}{ }^{H}$ - measurable contingent claim $F \in L^{2}(P)$ is given by 


$$
F(t)=e^{-r(T-t)} \widetilde{E}_{t}[F]
$$

Proof:

Since the market is complete there is a replicating portfolio of the claim $(m(t), s(t))$ whose value is:

and

$$
F(t)=m(t) M(t)+s(t) S(t)
$$

$F(T)=F$

We have that

$$
\begin{aligned}
d F(t) & =m(t) d M(t)+s(t) d S(t) \\
& =r F(t) d t+\sigma s(t) S(t) d B_{H}(t)
\end{aligned}
$$

By multiplying with $e^{-r t}$ and integrating it follows that

$$
e^{-r t} F(t)=F(0)+\int_{0}^{t} e^{-r \tau} \sigma s(\tau) S(\tau) d B_{H}(\tau), 0 \leq t \leq T
$$

By the fractional Clark-Ocone theorem (theorem 4.15 in $\mathrm{Hu}$ and Oksendal, 2003) we have that

$$
e^{-r T} F=E\left[e^{-r T} F\right]+e^{-r T} \int_{0}^{T} \widetilde{E}_{\tau}\left[D_{\tau} F\right] d B_{H}(\tau)
$$

where $D_{\tau} F$ is the Malliavin derivative of $F$ (definition 4.3 in $\mathrm{Hu}$ and Oksendal, 2003).

From the completeness of the market we get

$$
\widetilde{E}_{\tau}\left[D_{\tau} F\right]=e^{r(T-\tau)} \sigma s(\tau) S(\tau), 0 \leq \tau \leq T
$$

So we have that

$$
e^{-r T} F=E\left[e^{-r T} F\right]+\int_{0}^{T} e^{-r \tau} \sigma s(\tau) S(\tau) d B_{H}(\tau)
$$

It follows that

$$
\widetilde{E}_{t}\left[e^{-r T} F\right]=E\left[e^{-r T} F\right]+\widetilde{E}_{t}\left[\int_{0}^{T} e^{-r \tau} \sigma s(\tau) S(\tau) d B_{H}(\tau)\right]
$$

Using the fact that $\int_{0}^{t} e^{-r \tau} \sigma s(\tau) S(\tau) d B_{H}(\tau)$ is a quasi-martingale we have: 


$$
\widetilde{E}_{t}\left[e^{-r T} F\right]=E\left[e^{-r T} F\right]+\int_{0}^{t} e^{-r \tau} \sigma s(\tau) S(\tau) d B_{H}(\tau)
$$

From 3.1 and 3.2 we have that

$$
F(t)=e^{-r(T-t)} \widetilde{E}_{t}[F]
$$

q.e.d.

THEOREM 3.2 (fractional fundamental evaluation equation)

The price of a derivative on the stock price with a bounded payoff $f(S(T))$ is given by $F(t, S(t))$, where $F(t, S)$ is the solution of the PDE:

$$
\begin{aligned}
& \frac{\partial F}{\partial t}+H \sigma^{2} t^{2 H-1} S^{2} \frac{\partial^{2} F}{\partial S^{2}}+r S \frac{\partial F}{\partial S}-r F=0 \\
& F(T, S)=f(S)
\end{aligned}
$$

\section{Proof:}

From Theorem 3.1 and Theorem 2.2 it follows that the price at a moment $t$ of the derivative with payoff $f(S(T))$ is a function of $t$ and $S(t)$.

As in the classical Black-Scholes model (Black and Scholes, 1973) we consider a portfolio that contains a derivative and $-\Delta$ stock.

The value of this portfolio is

$$
\Pi(t)=F(t, S(t))-\Delta S(t)
$$

Under the market measure $\bar{P}$ using the fractional Ito formula (theorem 4.5 in Duncan, Hu and Pasik-Duncan, 2000) and the fact that

$$
\begin{aligned}
D_{\tau} S(\tau) & =S(\tau) D_{u}\left(\sigma B_{H}(\tau)+\mu \tau-\frac{\sigma^{2}}{2} \tau^{2 H}\right) \\
& =S(\tau) D_{u}\left(\sigma B_{H}(\tau)\right) \\
& =\sigma S(\tau) \mathbb{1}_{[0, \tau]}(u)
\end{aligned}
$$

and

$$
D_{\tau}^{\phi} S(\tau)=\sigma S(\tau) \int_{0}^{\tau} \phi(\tau, u) d u=\sigma H S(\tau) \tau^{2 H-1}
$$

we get that

$d \Pi(t)=d F(t, S(t))-\Delta d S(t)$ 


$$
=\left(\frac{\partial F}{\partial t}+H \sigma^{2} t^{2 H-1} S^{2} \frac{\partial^{2} F}{\partial S^{2}}+\mu S \frac{\partial F}{\partial S}-\Delta \mu S\right) d t+\left(\sigma S \frac{\partial F}{\partial S}-\Delta \sigma S\right) d \bar{B}_{H}(t)
$$

We want this portfolio to be riskless. So

$$
\Delta=\frac{\partial F}{\partial S} \quad \text { and } \quad d \Pi(t)=r \Pi(t) d t
$$

It follows that the evaluation equation is given by:

$$
\frac{\partial F}{\partial t}+r S \frac{\partial F}{\partial S}+H \sigma^{2} t^{2 H-1} S^{2} \frac{\partial^{2} F}{\partial S^{2}}-r F=0
$$

q.e.d.

\section{CONCLUSION}

In this paper it was developed a framework for evaluating derivatives if the underlying of the derivative contract is supposed to be driven by a fractional Brownian motion with Hurst parameter greater than 0.5.

We proved that in the fractional Black-Scholes market one can use the risk-neutral evaluation methodology but by using the quasi-conditional expectation. Thus, in this context, the price of a contingent claim is the quasiconditional expectation of the present value of the future cash-flows generated by this financial product.

We also obtained for the fractional Black-Scholes market the fundamental evaluation equation of a contingent claim. As in the classical Black-Scholes model the fundamental equation does not depend on the expected return $(\mu)$, but on the riskless interest rate $(r)$.

The risk-neutral evaluation methodology or the fundamental evaluation equation can be used to price a large class of derivatives in the context of fractional Black-Scholes market. For example, the price of an European call option with strike price $K$ cab be obtained by solving the PDE in Theorem 3.3 with the boundary condition $F(T, S)=\max (S-K, 0)$. 


\section{REFERENCES}

[1] Alos, E., O. Mazet and D. Nualart, (2001), "Stochastic calculus with respect to Gaussian processes”, Ann. Probab., 29, 766-801.

[2] Alos, E., O. Mazet and D. Nualart, (2000), "Stochastic calculus with respect to the fractional Brownian motion". Stochastic Processes and their Applications, 86, 1, 121-139.

[3] Bender, C., (2002), "The Fractional Ito Integral, Change of Measure and Absence of Arbitrage", University of Konstanz Working Paper, http://www.math.unikonstanz.de/ kohlmann/ftp/dp02 03.pdf

[4] Bender, C., (2004), "Explicit Solutions of a Class of Linear Fractional BSDEs", Systems \& Control Letters, 54, 7, 671-680.

[5] Black F. and M. Scholes, (1973), "The pricing of options and corporate liabilities", Journal of Political Economy, 81, 637-659.

[6] Decreusefond L. and A.S. Ustunel, (1999), "Stochastic analysis of the fractional Brownian motion”, Potential Anal., 10, 177-214.

[7] Duncan, T. E., Y. Hu and B. Pasik-Duncan, (2000), "Stochastic calculus for fractional Brownian motion, I. Theory", SIAM J. Control Optim., 38, 582-612.

[8] Hu, Y. and B. Oksendal, (2003), "Fractional white noise calculus and application to Finance”, Infin. Dimens. Anal. Quantum Probab. Relat. Top., 6, 1-32.

[9] Hu, Y., B. Oksendal, and A. Sulem, (2003), "Optimal consumption and portfolio in a Black-Scholes market driven by fractional Brownian motion”, Infin. Dimens. Anal. Quantum Probab. Relat. Top., 6, 519-536.

[10] Lin, S.J., (1995), "Stochastic analysis of fractional Brownian motion, fractional noises and applications", SIAM Review, 10, 422-437.

[11]Necula, C., (2002), "Option Pricing in a Fractional Brownian Motion Environment”, Advances in Economic and Financial Research - DOFIN Working Paper Series, DOFIN WP 2/2008, SSRN id 1286833

[12] Necula, C., (2003), "Barrier Options and a Reflection Principle of the Fractional Brownian Motion”. Advances in Economic and Financial Research - DOFIN Working Paper Series, DOFIN WP 6/2008, SSRN id 1286834

[13] Oksendal, B., (2003), "Fractional Brownian motion in finance", University of Oslo Working Paper, 28, http://www.math.uio.no/eprint/pure math/2003/28-03.pdf.

[14] Perez-Abreau V. and C. Tudor, (2002), "A Transfer Principle for Multiple Stochastic Fractional Integrals", CIMAT Working Paper, 1-01-03

[15] Rogers, L.C.G., (1997), "Arbitrage with fractional Brownian motion”, Math. Finance, 7, 95-105.

[16] Tudor, C., (2002), "On the Wiener integral with respect to the fractional Brownian motion”, Bol. Mex. Mat. Soc., 8, 97-106 